\title{
Neuwahl des DeGEval-Vorstandes 2019
}

Auf der Mitgliederversammlung am 11. September 2019 wurde ein neuer DeGEvalVorstand gewählt. Im Folgenden stellen sich die Mitglieder des neu gewählten Vorstandes vor.

Jan Ulrich Hense (Vorstandsvorsitzender, Dr. phil)

Jan Hense ist seit Januar 2014 Inhaber der Professur für Hochschuldidaktik und Evaluation am Fachbereich Psychologie der Justus-Liebig-Universität Gießen. Seine Arbeitsschwerpunkte liegen im Bereich der Gestaltung von Lehr- und Lernprozessen an der Hochschule sowie der Evaluation und Qualitätssicherung, insbesondere im Hochschulbereich. Sein besonderes Interesse gilt dabei der Frage, wie Evaluationsverfahren so zu gestalten sind, dass sie effektiv zur Qualitätsentwicklung der Lehre und anderer Gegenstandsbereiche beitragen können. $\mathrm{Zu}$ seinen weiteren Forschungsthemen gehören die Implementation innovativer Lehr-Lern-Formen in der Hochschullehre, Lernen mit digitalen Medien sowie Game-Based Learning und Gamification. Im Rahmen seiner akademischen Tätigkeit war Jan Hense an mehr als 30 Evaluationsprojekten von kommunaler bis zu Europäischer Ebene sowohl in Forschungs- als auch Auftragskontexten operativ und/oder leitend beteiligt. Als Gastdozent ist er an der Universität Bern in den Studiengängen Master/Diploma of Advanced Studies in Evaluation für das Thema „Programmtheorien in der Evaluation“ verantwortlich.

Fachpolitisch ist Jan Hense vor allem in der Gesellschaft für Evaluation (DeGEval e.V.) aktiv, wo er seit 2003 Mitglied ist und von 2008 bis 2015 Sprecher des Arbeitskreises ,Aus- und Weiterbildung in der Evaluation' war. In dieser Funktion lagen ihm vor allem die Professionalisierung der Evaluation sowie der politikfeldübergreifende Austausch über Evaluation am Herzen. Er war lokaler Ausrichter der DeGEval-Jahrestagung 2013 in München und von 2013 bis 2016, seit 2015 in leitender Funktion, Mitglied der DeGEval-Arbeitsgruppe zur Revision der „Standards für Evaluation".

Jan Hense ist seit 2015 Vorstandsmitglied und seit 2017 Vorstandsvorsitzender der DeGEval - Gesellschaft für Evaluation e.V. 
Marianne Lück-Filsinger (stv. Vorstandsvorsitzende, Dr. ${ }^{\text {in }}$ )

Marianne Lück-Filsinger studierte, nach einer Ausbildung als Chemielaborantin bei der BASF in Ludwigshafen, an den Pädagogischen Hochschulen Heidelberg und Rheinland, Abt. Neuss für das Lehramt Sekundarstufe I (Chemie und kath. Theologie).

Nach der ersten Staatsprüfung nahm sie ein Studium der Soziologie, Erziehungswissenschaft und Kath. Theologie an der Westfälischen Wilhelms-Universität Münster auf und schloss dieses mit einem Magister Artium ab. Promoviert hat sie an der Johannes-Gutenberg-Universität in Mainz 2006 mit einer qualitativ-empirischen Studie „Kinder- und Jugendberichterstattung in den Bundesländern“.

Marianne Lück-Filsinger hat in den letzten 20 Jahren verschiedene Forschungs-, Evaluations- und Entwicklungsprojekte durchgeführt, insbesondere Projekte der (stadtteilbezogenen) Jugendarbeit, der kommunalen Jugendpolitik, der Schulsozialarbeit, der Stadt-, Stadtteil- bzw. Quartiersentwicklung und der Arbeitsmarkt- bzw. Integrationspolitik. Seit 2009 ist sie Leiterin der Forschungsgruppe ,Bildungs-, Evaluations- und Sozialstudien' (ForBES) an der Fakultät für Sozialwissenschaften der Hochschule für Technik und Wirtschaft des Saarlandes (HTW).

Seit mehr als 20 Jahren ist sie als Lehrbeauftragte an verschiedenen Hochschulen tätig. Seit mehr als 10 Jahren liegt der Schwerpunkt der Lehrtätigkeit bei den ,Qualitativen Datenerhebungs- und Auswertungsmethoden“ im Bachelorstudiengang Soziale Arbeit und Pädagogik der Kindheit und im Masterstudiengang Evaluation an der Universität des Saarlandes in Kooperation mit der HTW des Saarlandes.

Marianne Lück-Filsinger ist seit 2009 in der DeGEval engagiert, in der ersten Zeit vor allem im Arbeitskreis ,Soziale Dienstleistungen'. Sie war in der Zeit von 2010 bis 2014 eine der Sprecher(innen) des Arbeitskreises Soziale Dienstleistungen und hat seit 2015 im Programmkomitee für die Jahrestagungen mitgearbeitet. In den letzten beiden Jahren hat sie zusammen mit einer Kollegin aus dem Kreis des Arbeitskreises in einem Tandem das Programmkomitee koordiniert.

Marianne Lück-Filsinger ist seit 2017 Vorstandsmitglied und seit 2019 stellvertretende Vorsitzende der DeGEval - Gesellschaft für Evaluation e.V.

Sonja Kind (Vorstandsmitglied, Dipl. Biol., Dr. ${ }^{\text {in }}$ )

Sonja Kind studierte Biologie mit Schwerpunkt Molekularbiologie an der Universität zu Köln und schloss ihre Diplomarbeit 1999 ab. Im Anschluss promovierte sie im Fach Wirtschafts- und Sozialwissenschaften innerhalb des interdisziplinären und Entrepreneurship-orientierten Stipendiatenprogramms EXIST-HighTEPP an der Universität Bamberg. Von 2004 bis 2005 arbeitete sie als Beraterin in einer Strategieberatung.

Seit 2005 ist Frau Kind Mitarbeiterin bei der VDI/VDE-IT und war bereits in verschiedenen Bereichen des Hauses tätig. Einer ihrer wesentlichen Arbeitsschwerpunkte besteht in der Evaluation von Forschungs-, Technologie- und Innovationsprogrammen. Seit 2010 ist sie Sprecherin des Themenfelds Evaluation im Institut für Innovation und Technik (iit) innerhalb der VDI/VDE-IT. Frau Kind hat zahlreiche Programm- und Projektevaluationen für öffentliche Auftraggebende durchgeführt, insbesondere für Bundes- und Landesministerien. Das thematische Spektrum 
reicht von Clusterpolitik, IuK über Elektromobilität bis hin zur Gründungsunterstützung und Mittelstandsförderung.

Seit einigen Jahren ist die Innovations- und Technikanalyse ein weiterer Fokus ihrer Arbeit. Frau Kind arbeitet seit Februar 2014 im Projekt zur Durchführung eines Horizon-Scannings für das TAB - Büro für Technikfolgenabschätzung beim Deutschen Bundestag, dessen Leitung sie 2016 übernommen hat.

In den Jahren 2015 bis 2017 hat Frau Kind eine Ausbildung zum Systemischen Coach und Organisationsberaterin am artop-Institut der Berliner Humboldt-Universität absolviert und ist nebenberuflich als Coach und Organisationsberaterin tätig.

Frau Kind engagiert sich seit 2006 in der DeGEval und war dort u.a. bis 2018 Sprecherin des Arbeitskreises ,Evaluation in der Wirtschaft'. Sie hat an der Erarbeitung des Leitfadens „Evaluation in der Wirtschaft“ mitgewirkt und hat von 2012 bis 2013 die Kassenprüfung für den Verein übernommen.

Sonja Kind ist seit 2019 Mitglied des Vorstands der DeGEval - Gesellschaft für Evaluation e.V.

Manfred Rolfes (Vorstandsmitglied, Dr.)

Manfred Rolfes studierte von 1982 bis 1988 an der Universität Osnabrück Geographie, Germanistik und Erziehungswissenschaften. Als wissenschaftlicher Mitarbeiter war er von 1989 bis 2003 am Fachgebiet Geographie der Universität Osnabrück sowie am Wissenschaftlichen Zentrum für Berufs- und Hochschulforschung der Gesamthochschule Kassel (heute: International Centre for Higher Education Research Kassel - INCHER) tätig. Seit 2003 ist er Professor für Angewandte Humangeographie und Regionalwissenschaften am Institut für Umweltwissenschaften und Geographie der Universität Potsdam und seit 2012 Lehrbeauftragter im Masterstudiengang Kriminologie und Polizeiwissenschaft an der Ruhr-Universität Bochum.

Seine Forschungsschwerpunkte liegen in den Themenfeldern Angewandte Stadtforschung; (Un-)Sicherheit, Risiken und Raum; Alltagstourismus im globalen Süden und der Evaluationsforschung. Manfred Rolfes kam über die (Selbst-)Evaluation von Studiengängen zur Evaluationsforschung. Seit 2000 beschäftigt er sich anwendungsbezogen mit der Evaluation von Stadt(teil)- und Regionalentwicklungsprozessen, entwickelte in einem interdisziplinären Team einen Leitfaden zur Evaluierung nationaler Städtebauförderungsprogramme und führt partizipativ angelegte qualitative Evaluationen von Präventionskonzepten durch (in den Handlungsfeldern Kriminalprävention und Auseinandersetzung mit Rechtsextremismus/Rechtspopulismus). Sein Blick auf Evaluationen, Bewertungen und Projekt- bzw. Programmwirkungen ist dabei gekennzeichnet von einem system(theoret)ischen Verständnis, das die Multiperspektivität und Kontextabhängigkeit in diesem Handlungsfeld explizit berücksichtigt.

Im Jahr 2001 wurde Manfred Rolfes Mitglied der Gesellschaft für Evaluation (DeGEval e. V.) und engagiert sich seit 2002 im Arbeitskreis ,Stadt- und Regionalentwicklung'. Von 2010 bis 2019 war er einer der Sprecher(innen) des Arbeitskreises.

Manfred Rolfes ist seit 2019 Mitglied des Vorstands der DeGEval - Gesellschaft für Evaluation e.V. 
Stefan Schmidt (Vorstandsmitglied, Diplom Sozialarbeiter, MA of Organizational Psychology)

Stefan Schmidt kam über die Praxis der Kinder- und Jugendhilfe zur Evaluation. Er studierte zunächst Sozialarbeit an der ev. Hochschule Rheinland-Westfalen-Lippe, schrieb seine Diplomarbeit 1996 zur Einführung der outputorientierten Steuerung in der Jugendhilfe und leitete ab 1998 ein städtisches Jugendzentrum in Bochum. Dort führte er mit seinem Team Zielklärungsprozesse durch und implementierte regelmäBige Selbstevaluationen. Den Master der Fachrichtung Arbeits- und Organisationspsychologie erwarb er im Jahr 2004 berufsbegleitend an der Fern Universität Hagen mit einer Arbeit über die Evaluation des Organisationsentwicklungsprozesses zur Zusammenlegung der Arbeits- und Sozialverwaltung im Zuge der Einführung des SGB II. Die Masterarbeit wurde im Rahmen eines Praxissemesters bei Univation (Köln) erstellt. Seit Ende 2004 ist Stefan Schmidt als selbständiger Evaluator tätig und führt das Unternehmen schmidt evaluation (Köln).

Der Schwerpunkt seiner Aktivitäten liegt auf der Durchführung von externen Programm- und Projektevaluationen für öffentliche Auftraggebende (Land, Bund, EU), Stiftungen und sonstige Träger. Ausgehend von der Kinder- und Jugendhilfe hat er sich nach und nach weitere Themen erschlossen und führt Evaluationen mittlerweile quer durch unterschiedliche Themenbereiche durch, u.a. Arbeitsmarkt, Bildung, Demokratie, Wirtschaft und Gesundheit/Ernährung. Für umfangreiche und mehrjährige Evaluationen kooperiert er projektbezogen, fach- und feldspezifisch mit verschiedenen Partner(inne)n. Ein weiteres Aktivitätsfeld ist die Beratung und Begleitung von regionalen und kommunalen Netzwerken in den Bereichen Bildung, Frühe Bildung und Integration. Er berät und qualifiziert Entscheidungsträger(innen), Leitungs- und Fachkräfte zu den Themen wirkungsorientierte Steuerung, interne Evaluation, Selbstevaluation und Qualitätsentwicklung.

An der TH Köln übte er von 2014 bis 2018 einen Lehrauftrag zum Qualitätsmanagement in pädagogischen Institutionen im Rahmen des Studiengangs BA Pädagogik der Kindheit und Familienbildung aus.

Stefan Schmidt ist seit 2004 Mitglied der DeGEval. Von 2012 bis 2016 fungierte er als einer der Sprecher(innen) des Arbeitskreises ,Soziale Dienstleistungen'. Seit dem Jahr 2017 ist er Mitglied des Vorstands der DeGEval - Gesellschaft für Evaluation e.V.

Angela Wroblewski (Vorstandsmitglied, Dr. ${ }^{\text {in }}$ )

Angela Wroblewski studierte neben einer Beschäftigung als Bankangestellte Soziologie an der Universität Wien und absolvierte von 1996 bis 1998 ein postgraduales Studium am Institut für höhere Studien (IHS) und ein Masterstudium in Social Science Data Analysis an der Universität Essex (UK).

Seit Ende der 1990er Jahre ist sie in Evaluationen von Maßnahmen der Frauenoder Gleichstellungsförderung in den Bereichen Arbeitsmarkt, Bildung und Wissenschaft eingebunden. In den letzten 15 Jahren hat sie sich überwiegend mit Gleichstellungspolitik im Bereich Wissenschaft und Forschung auseinandergesetzt. Derzeit koordiniert sie das EU-geförderte Projekt TARGET - Taking a Reflexive Approach to Gender Equality for institutional Transformation (2017-2021). Im Rahmen des 
Projekts werden sieben Forschungsinstitutionen bzw. Forschungsfördereinrichtungen aus Mittelmeerländern bei der Entwicklung, der Umsetzung und dem Monitoring von Gleichstellungsplänen unterstützt. Darüber hinaus ist sie in das ebenfalls EU-geförderte Projekt GENDERACTION eingebunden, wo sie für das Monitoring der Umsetzung nationaler Aktionspläne für Gleichstellung in Wissenschaft und Forschung verantwortlich ist.

Seit 2005 ist Angela Wroblewski in der DeGEval aktiv. Sie ist Gründungsmitglied des Arbeitskreises ,Gender Mainstreaming " und war bis 2019 eine seiner Sprecherinnen. Sie hat u.a. an den Genderkompetenzprofilen für Evaluator(inn)en mitgearbeitet. Sie war in den Jahren 2015 bis 2019 im Programmkomitee für die Jahrestagungen aktiv. Angela Wroblewski ist auch Mitglied der EES - European Evaluation Society und vertritt das IHS im Rahmen der fteval - der Plattform für Forschungs- und Technologieevaluierung.

Angela Wroblewski ist seit 2019 Mitglied des Vorstands der DeGEval - Gesellschaft für Evaluation e.V. 